\title{
Abnormal Persistence of Embryonic Blood Supply of Liver: Anatomist's Delight, Surgeon's Nightmare
}

\author{
Sabah Yaseen, Surbhi Wadhwa*, Kahkashan Jeelani, Anita Mahajan, Sabita Mishra
}

\begin{abstract}
The high incidence of hepato-biliary vascular anatomy variations necessitates its evaluation prior to performing liver transplantation, hepatobiliary, pancreatic, gastric and oesophageal surgeries. We report a unique case of persistence of embryonic arteries of the liver, wherein, the liver was supplied by five vessels. In addition to the usual right and left hepatic arteries from the hepatic artery proper, the liver received two accessory right hepatic arteries, one from the gastroduodenal artery, while another arising from superior mesenteric artery and an accessory left hepatic artery, from the left gastric artery. The origin of gastroduodenal artery was found to be unusually high and its abnormal anterior course over the common bile duct further added complexity to the hepatobiliary anatomy. The presence of these aberrant and accessory arteries predisposes to inadvertent injury leading to patient morbidity and sometimes mortality.
\end{abstract}

\section{KEYWORDS}

common hepatic artery; proper hepatic artery; accessory hepatic artery; gastroduodenal artery

\section{AUTHOR AFFILIATIONS}

Department of Anatomy, Maulana Azad Medical College, New Delhi, India

* Corresponding author: Department of Anatomy, Maulana Azad Medical College, New Delhi, 110002, India;

e-mail: wadhwa.surbhi@gmail.com

Received: 28 November 2018

Accepted: 27 March 2019

Published online: 26 July 2019

Acta Medica (Hradec Králové) 2019; 62(2): 72-76

https://doi.org/10.14712/18059694.2019.106

(c) 2019 The Authors. This is an open-access article distributed under the terms of the Creative Commons Attribution License (http://creativecommons.org/licenses/by/4.0), which permits unrestricted use, distribution, and reproduction in any medium, provided the original author and source are credited. 


\section{INTRODUCTION}

The celiac trunk (CT) arises from abdominal aorta at the level of 12th thoracic vertebra, passes almost horizontally forwards for 1 to $3 \mathrm{cms}$. It trifurcates into left gastric artery (LGA), common hepatic artery (CHA) and splenic artery (SA) (1). While the LGA and SA run towards the left to supply the esophagus and spleen respectively, the CHA passes antero-laterally above the superior border of pancreas to the right as far as the first part of duodenum (1). After the origin of gastroduodenal artery (GDA), CHA continues as the hepatic artery proper (HAP). The HAP runs within the free margin of lesser omentum, where it lies anterior to portal vein $(\mathrm{PV})$ and medial to common bile duct (CBD). It ends by dividing into right and left hepatic arteries at variable distance from the porta hepatis (1). Anatomical variations in the blood supply of liver and hepatic arteries are found in one third of the individuals $(2,3)$. The knowledge of variations in the origin of arteries supplying the liver is very important to perform surgeries like pancreaticoduodenectomy and liver transplant as well as during hepatic artery infusion chemotherapy (4). Due to occurrence of these variations it is imperative to map the individual specific arterial patterns by performing CT angiograms/ MR angiograms prior to undertaking major hepato-pancreatic-biliary surgical procedures (5). On table, during surgeries these variations increase the potential risk of complications like haemorrhage, liver necrosis, ischemia and are the foremost reasons for conversion of laparoscopic procedures to open surgery.

\section{CASE REPORT}

Routine abdominal dissection for undergraduate teaching in the Department of Anatomy at Maulana Azad Medical College, of 88 year old female with previous surgical history of right mastectomy only, revealed multiple variations in the branches of coeliac trunk and superior mesenteric artery. There were five hepatic arteries, each originating from a different source supplying the liver.

The CT had normal origin from the ventral aspect of abdominal aorta, at T12 vertebral level between aortic hiatus and the superior border of pancreas. Immediately after origin it gave rise to the right \& left inferior phrenic arteries (IPA). The IPA had usual course and distribution thereafter. While the right IPA ran posterior to the inferior vena cava, the left IPA passed behind the esophagus, to supply the respective side of diaphragm \& suprarenals. The left IPA also supplied the abdominal part of the esophagus (Fig. 1).

The CT branched in to usual three branches LGA, SA and CHA. CHA (length $4.5 \mathrm{~cm}$ ) ran upwards over the first part of duodenum and gave rise to GDA and continued as HAP. The origin of GDA was unusually higher, approximately $4 \mathrm{~cm}$ from the pylorus. The GDA then turned sharply downwards from its origin and was present on the left side of CBD initially. Further distally, it took a C shaped curve anterior to the $\mathrm{CBD}$, thereafter running posterior to the first part of duodenum where it divided into right gastroepiploic and anterior pancreatic duodenal artery. There was presence of pancreatic tissue between the GDA and

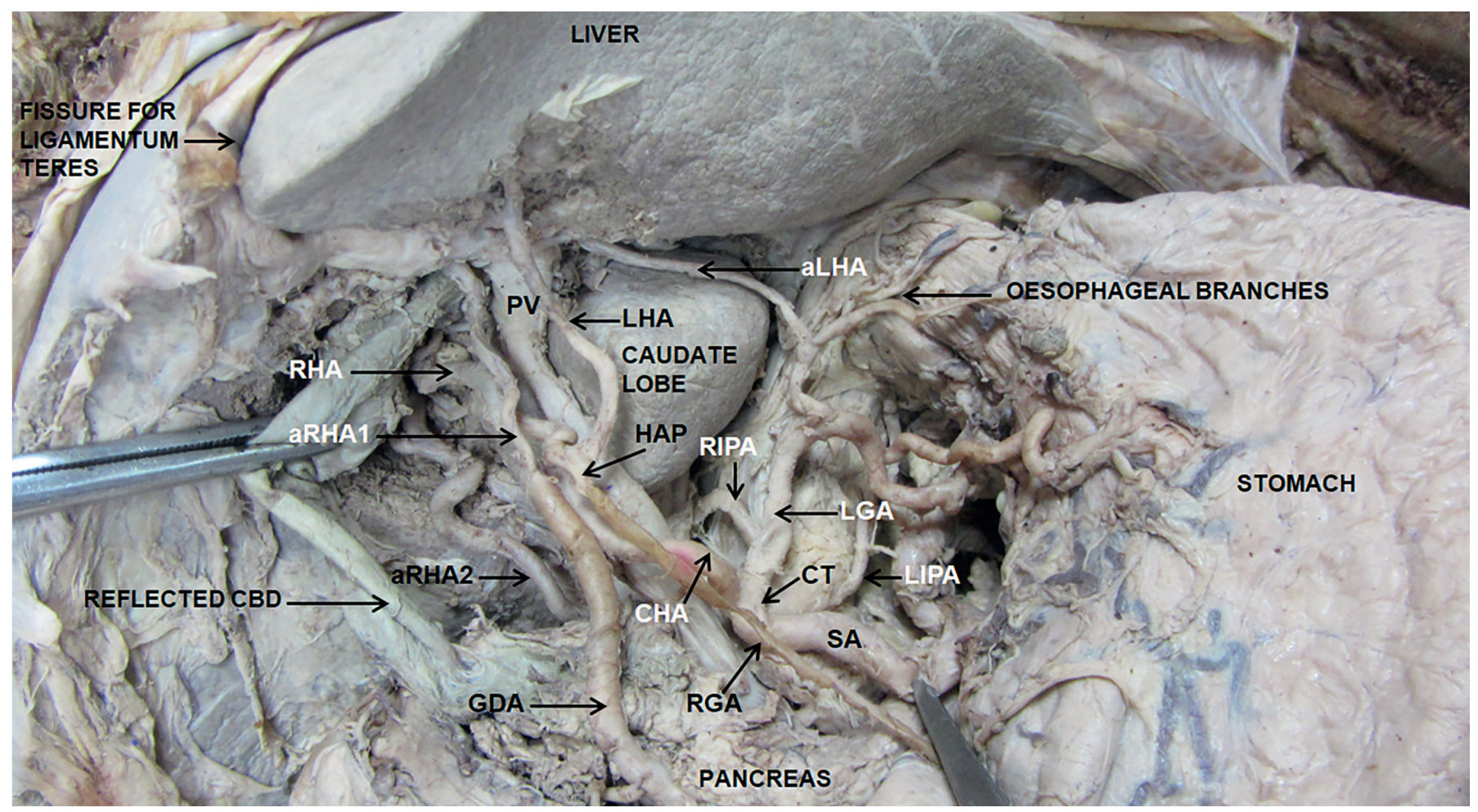

Fig. 1 A dissected specimen of abdomen: The celiac trunk (CT) dividing into three branches - Left gastric artery (LGA), Splenic artery (SA) and Common hepatic artery (CHA). Right inferior phrenic artery (RIPA) and Left inferior phrenic artery (LIPA) also originating from CT. CHA gives rise to high rising Gastroduodenal artery (GDA) and a small stump of Hepatic artery proper (HAP). HAP divides into Right hepatic artery (RHA) and Left hepatic artery (LHA). LGA gives rise to Accessory left hepatic artery (aLHA). The Common Bile Duct (CBD) has been retracted to clearly visualise One Accessory right hepatic artery (aRHA1) arising from GDA while another Accessory right hepatic artery (aRHA2) arising from superior mesenteric artery. 


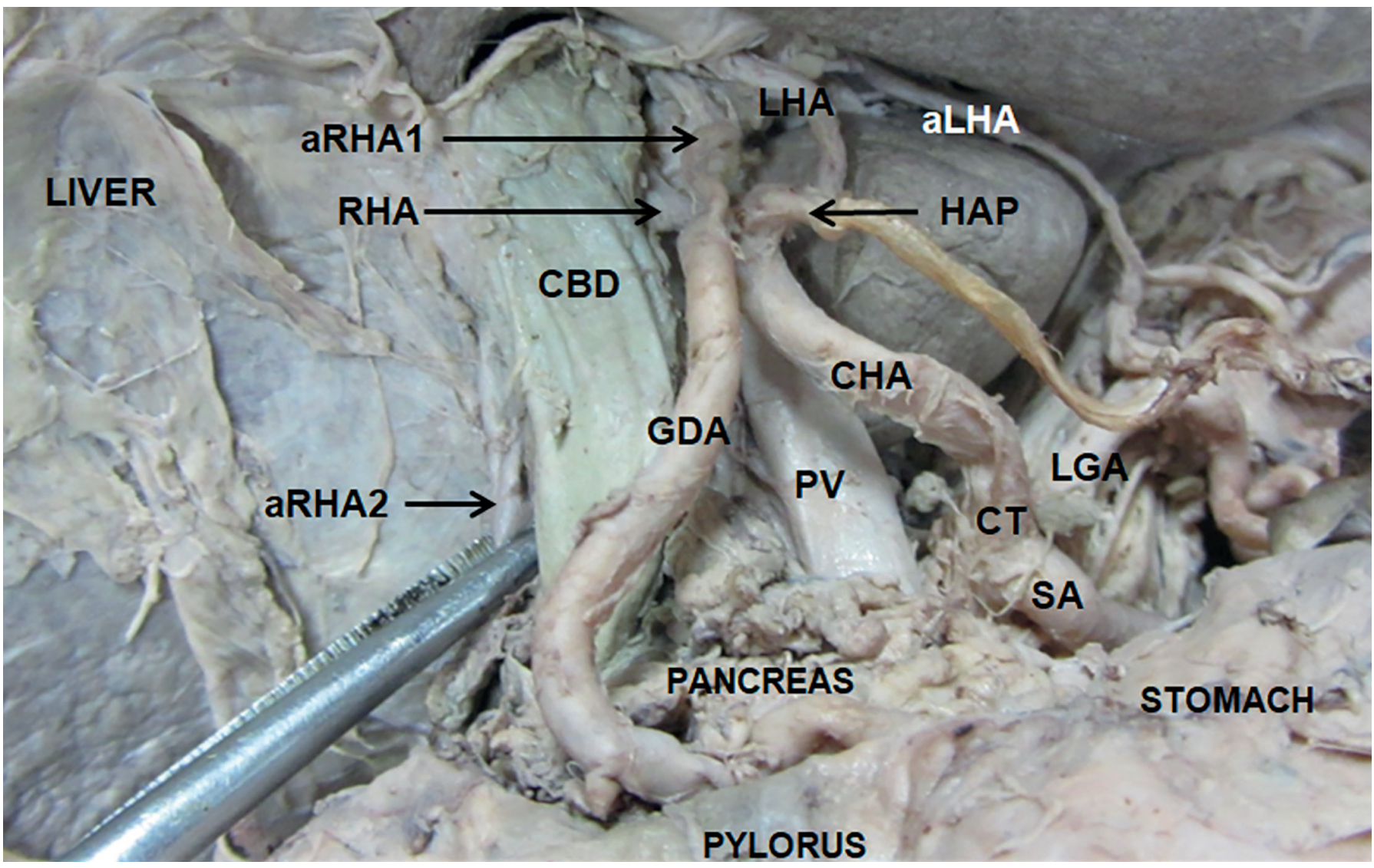

Fig. 2 Depicting High origin of gastroduodenal artery (GDA) and relation of GDA and accessory right hepatic artery (aRHA2) with common bile duct (CBD). Note the $\mathrm{C}$ shaped mid and distal portion of GDA present anterior to the CBD with a small portion of pancreas intervening in between.

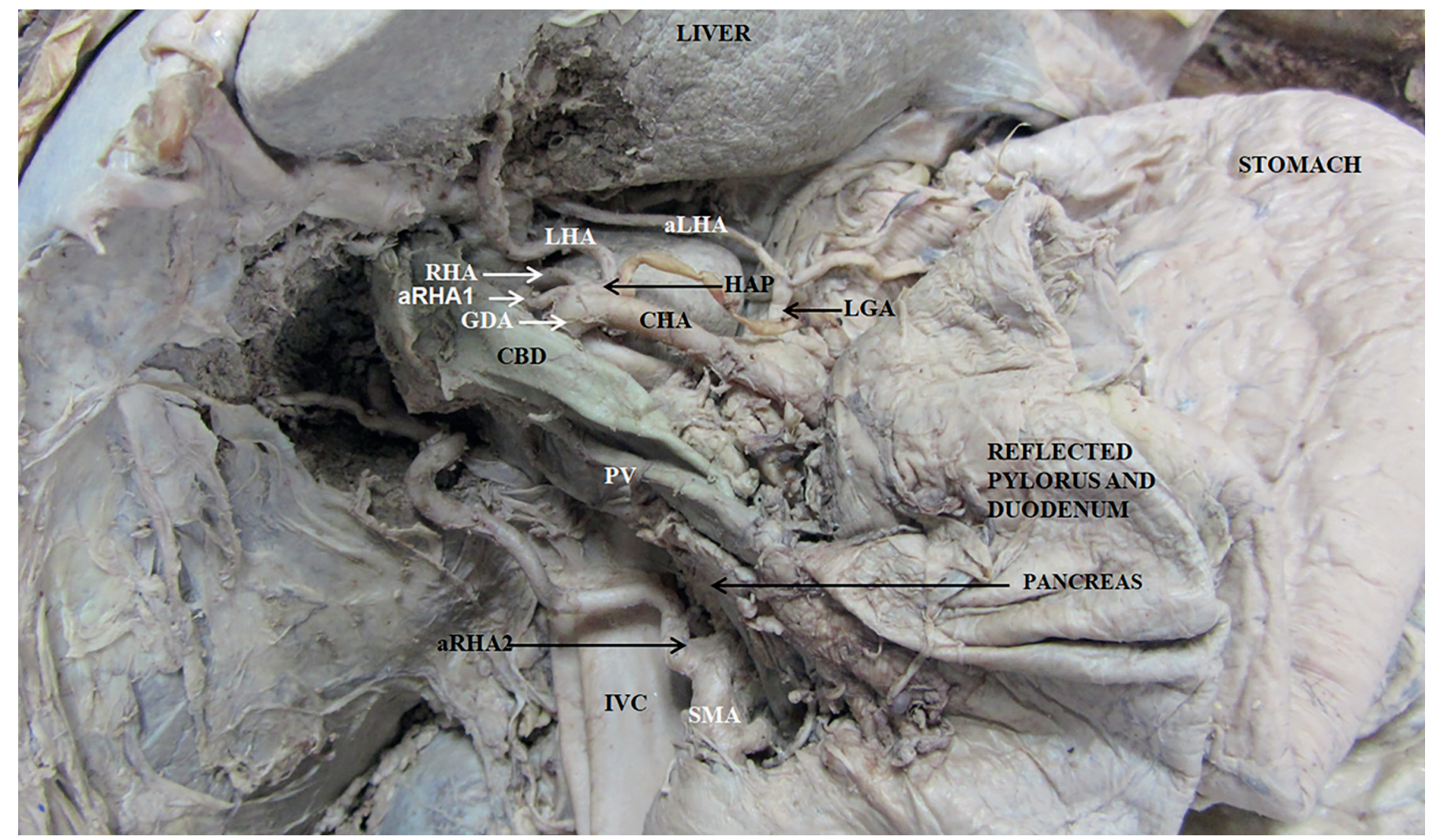

Fig. 3 Showing origin of accessory right hepatic artery (aRHA2) from superior mesenteric artery (SMA) behind the head of pancreas. 
CBD only near the pylorus. The first accessory right hepatic artery (aRHAl) arose from the GDA where it turned sharply to descend to the left of CBD (Fig. 1, Fig. 2).

HAP was present as a small stump $(0.4 \mathrm{~cm})$. It gave rise to the right gastric artery (RGA) proximally and then bifurcated into the right hepatic artery (RHA) and left hepatic artery (LHA). The RHA passed obliquely upwards for $3 \mathrm{~cm}$, anterior to the PV and posteriomedial to hepatic duct to enter into porta hepatis. It was crossed anteriorly by aRHAl from right to left (Fig. 1, Fig. 2). Another accessory right hepatic artery (aRHA2) was seen arising from SMA posterior to the pancreas. It travelled posterior to the CBD and to the right of aRHAl (Fig. 3). LHA ascended for about $2.4 \mathrm{~cm}$ to enter the porta hepatis, on the left side of fissure for ligamentum teres and supplied the left lobe of liver. There was an accessory left hepatic artery (aLHA), a branch of LGA, which entered the anatomical left lobe of the liver medial to the PV (Fig. 1, Fig. 2). Thus at the porta hepatis LHA and aRHA1 were to the left of the bile duct, while RHA and aRHA2 were posterior to it. Posterior most structure was the portal vein. RHA and aRHA2 were lateral to it and aLHA entered the liver medial to PV. Near the superior border of the pylorus and first part of duodenum, the CBD was sandwiched between GDA anteriorly and aRHA2 posteriorly (Fig. 2).

\section{DISCUSSION}

Embryologically there are three lobes during development of mammalian liver - right lateral, central and left lateral lobes. Each of these has its own embryonic arteries supplying it. The left hepatic artery arises from the LGA and supplies the developing left lateral part of the liver. The right lateral anlage is supplied by a branch arising from SMA and the central lobe by the proper hepatic artery, arising from the junction of CHA and GDA, branches of CT. These arteries anastomose in the developing liver to supply the viscera. During the early embryonic stages, the developing liver anlage is larger than the developing gut. Later on the size of the liver proportionately decreases while that of the gut increases. Also the enlarging stomach and the spleen further reduce the size of the left lobe. These rearrangements cause regression and shifting of these three embryonal arterial roots results in the final single hepatic artery becoming the predominant artery supplying the liver. However variable persistence/absorption of the vessels result in anomalous vasculature (6). In the present case liver was supplied by five branches - the usual, RHA and LHA branches of a small stump PHA and additional aRHA1, branch of GDA, aRHA2 arising from SMA and aLHA, branch of LGA. Thus, all three embryonal arterial roots persisted as aRHA2, aLHA and middle vessel as PHA (although a short one) and supplied the liver (6) (Fig. 4). However this fails to explain the presence of an aRHA1 from GDA and the IPAs arising from the CT. These could be the remnant one of the branches of Tandlers longitudinal anastomosis which develops between the ventral abdominal aortic branches supplying the gut (7) (Fig. 4). Not only were accessory and aberrant branches present, the course of these vessels was also variable. The GDA branched out from the CHA higher than usual $-4 \mathrm{~cm}$ above the pylorus. At the porta hepatis LHA and aRHA1 were to the left of the bile duct, while RHA and aRHA2 were posterior to it. In its lower part the GDA took a $\mathrm{C}$ shaped curved bend over the CBD.

Michel's classification for hepatic artery describes a number of variants. However the present case does not fall under any of the described variations. It is similar to the type VII variant which has an incidence of $1 \%$, in which accessory right and left hepatic arteries arise from SMA and

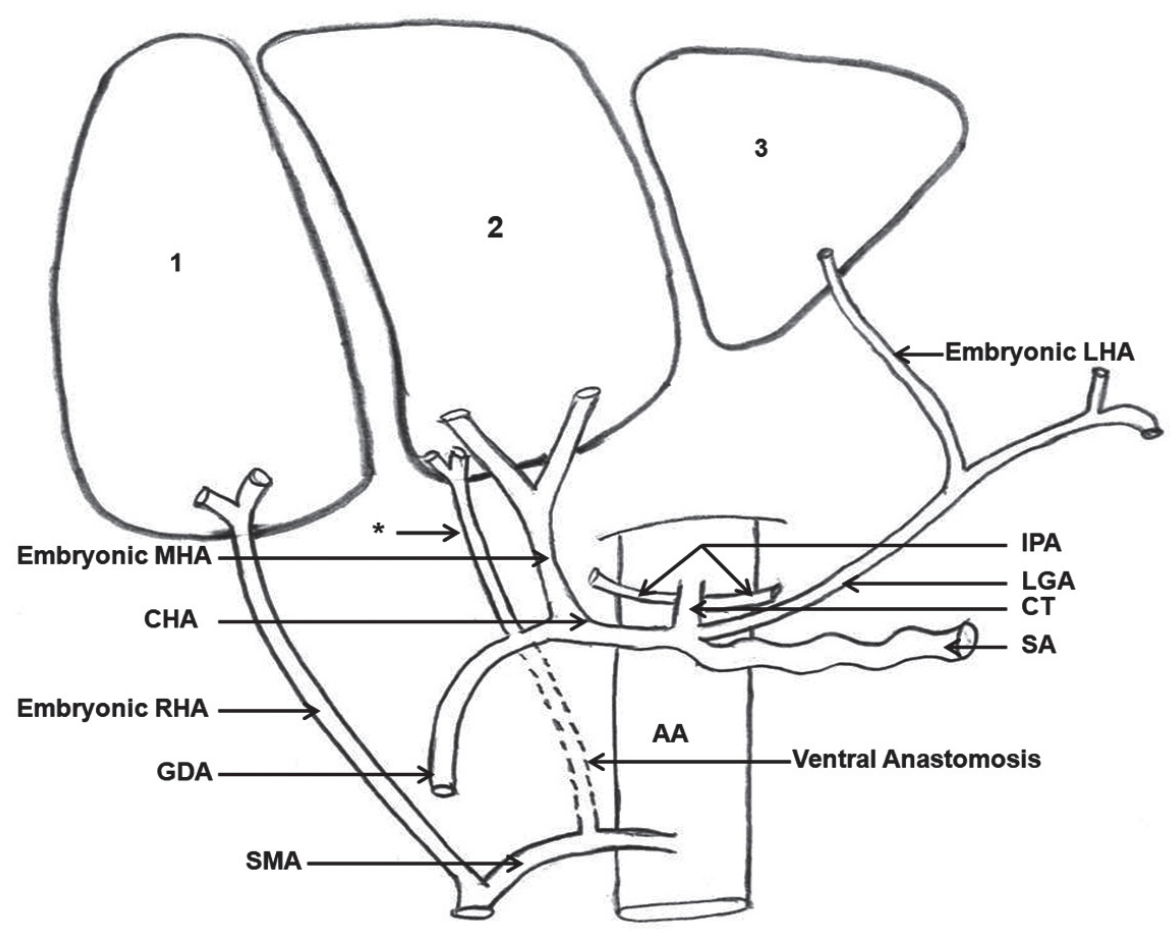

Fig. 4 Diagrammatic representation of the blood supply of the liver. 1, 2 and 3 are the right lateral, central and left lateral lobes of the developing liver. Note the embryonic right hepatic artery (RHA) is a branch of superior mesenteric artery (SMA), middle hepatic artery (MHA), a branch from the junction of common hepatic artery (CHA) and gastroduodenal artery (GDA) and left hepatic artery (LHA) a branch of left gastric artery (LGA). Superimposed are the ventral anastomosis between branches of celiac trunk (CT) and SMA, which may have persisted in this case as accessory right hepatic artery 1 (aRHA1) (*) to supply the liver. $A A$-abdominal aorta, SA-splenic artery, IPA-inferior phrenic artery. 
LGA respectively. However there is no mention of an aRHA from GDA in any of the variations described by Michel (2). A computed tomography study of CT was done by Osman et al, which revealed the classical trifurcation of CT in $63.8 \%$ cases (8). In present case, the CT trifurcated classically into LGA, SA and CHA however it also gave origin to both IPA which has been reported by other authors (1).

The mean distance between GDA and pylorus is usually $2.8 \mathrm{~cm}$ with a range of $1-5 \mathrm{~cm}$. In the present case the GDA was $4 \mathrm{~cm}$ superior to the pylorus hence a high rising artery. Additionally it was coursing anterior to the mid and distal portion of the CBD. Such a course variation is extremely rare as reported by Prudhomme et al. (9). However this is of significance as injury to GDA during laproscopic / open CBD exploration for impacted choledocholithiasis could result in severe haemorrhaging. The GDA could also get injured inadvertently during extension of choledochotomy or while repairing the choledochotomy, getting included in suture bite. The posterior wall of first part of duodenum is the most common site of duodenal ulcer and haemorrhage in these cases is reported to occur in 15-25\% cases. The GDA because of its location is the most commonly involved artery. Haemostasis of the bleeding artery and ulcer involves suture ligating the involved artery. The attempt to suture or ligate in the present case can potentially risk injury to underlying CBD. The close relationship between the GDA and CBD although seen rarely, necessitates that before ligation the surgeon verifies the position of the duct either by on table cholangiography, cholecystocopy or by inserting a probe in the duct (9). Lopez-Andujar et al. in 2007 reported concomitant existence of aLHA from LGA and aRHA from SMA with associated presence of LHA and RHA from PHA in only $0.6 \%$ cases. In none of the 12 variants reported by them did they find an accessory branch from GDA (3). aRHA1 originating from GDA is another rare though reported finding present in this case. Futara et al. observed such accessory vessel in only $1-2 \%$ cases (10). The presence of this vessel at the point where GDA sprouts and turns from its parent CHA could further complicate the retrograde introduction of catheter into the PHA since the PHA and aRHAl are originating in close proximity to each other and the length of PHA is unusually short. The presence of other accessory hepatic arteries like aRHA2 and aLHA as in present case may lead to inadequate perfusion of the liver parenchyma with chemotherapeutic drugs.

When aberrant arteries are encountered intraoperatively or are not recognized promptly, there is a higher risk of vascular injury. Commonly the aRHA arise from SMA both during cadaveric dissection and intraoperatively with an incidence of 11-26.5\% (11). Pancreaticoduodenectomy is the procedure of choice in patients with pancreatic or periampullary tumour and hence the presence of aberrant or replaced aRHA increase the rate of morbidity (11). An aRHA may leads to an alteration in the surgical approach and can also adversely affect the outcomes of the surgical procedure. Excessive handling of these vessels intraoperatively further damages the adventitia of the vessels predisposing to increase risk of pseudoaneurysm (12, 13). Unnecessary retraction of the pancreatic head should also be avoided in such instances as it increases the chances of thrombosis of the aberrant vessels.
During liver transplantation, it is extremely important to understand the complete anatomy and possible variations of liver. Arterial reconstruction is an important step during transplant surgeries. To obtain an optimal perfusion of the graft all the accessory arteries should be recognised at the time of organ collection and reconstructed as insufficient blood supply can lead to graft loss (14). Hepatic artery variations can increase postoperative morbidity, such as bleeding, ischemia or stenosis of the biliary anastomosis, hepatic abscess and hepatic infarction (15).

\section{CONCLUSION}

The anatomical variations in the celiac axis and hepatic artery are numerous. This case report identifies the variation in both. The unique findings of this case are the simultaneous existence of accessory branches from LGA, GDA and SMA. The accessory branch from GDA is rare finding. Through knowledge of these arterial pattern are of immense importance in preoperative planning, all surgical and interventional radiological procedures involving upper abdomen as well as hepatobiliary surgeries.

\section{REFERENCES}

1. Standring $\mathrm{S}$. The anatomical basis of clinical practice. 41st ed. New York: Elsevier, 2016.

2. Michels NA. Newer anatomy of the liver and its variant blood supply and collateral circulation. Am J Surg 1966; 112: 337-47.

3. Lopez AR, Moya A, Montalva E, Berenguer M, et al. Lesson learned from anatomic variants of Hepatic Artery in 1,081 Transplanted livers. Liver Transpl 2007; 13: 1401-4.

4. Wadhwa S, Khorwal G, Tigga SR. Retroportal proper hepatic artery with malrotated gut. Anat Sci Int 2013; 88(4): 242-5.

5. Konstantinos N, Maria P, Nikolaos L, Georgios K, Stylianos A. The coexistence of both replaced proper hepatic and gastroduodenal arteries due to common hepatic artery absence. Surg Radiol Anat 2017; 39: 1293-6.

6. Couinaud C. Surgical anatomy of the liver revisited: Embryology. Paris: Couinaud, 1989

7. Tandler J. Uber die Varietaten der Arteriacoeliaca und deren Entwickelung. Anat Hft 1904; 25: 473-500.

8. Osman AM, Abdrabou A. Celiac trunk and hepatic artery variants: A retrospective preliminary MSCT report among Egyptian patients. The Egyptian Journal of Radiology and Nuclear Medicine 2016; 47: 1451-8.

9. Prudhomme M, Canovas F, Godleswki G, Bonnel F. The relationship of the bile duct and retroduodenal arteries and their importance in the surgical treatment of hemorrhagic duodenal ulcers. Surg Radiol Anat 1997; 19: 227-30.

10. Futara G, Ali A, Kinfu Y. Variations of the hepatic and cystic arteries among Ethiopians. Ethiop Med J 2001; 39: 133-42.

11. Eshuis WJ, Loohuis KM, Busch ORC, Gulik TM, Gouma DJ. Influence of aberrant right hepatic artery on perioperative course and longterm survival after pancreatoduodenectomy. HPB 2011; 13: 161-7.

12. Rammohan A, Palaniappan R, Pitchaimuthu A, Rajendran K, Perumal SK, Balaraman K. Implications of the presence of an aberrant right hepatic artery in patients undergoing pancreaticoduodenectomy. World J Gastrointest Surg 2014; 6(1): 9-13.

13. Kosai NR, Reynu R, Abdikarim M, et al. Multiple Acute Aortic Dissection in a Young Adult. Med \& Health 2014; $9(2): 143-9$.

14. Michał P, Hogendorf P, Topol M. Variations of the hepatobiliary vasculature including coexistence of accessory right hepatic artery with unusually arising double cystic arteries: case report and literature review. Anat Sci Int 2014; 89: 195-8.

15. Sebben GA, Rocha SL, Sebben MA, Filho RP, Goncalves BH. Variations of hepatic artery: anatomical study on cadavers. HPB 2016; 18 : $580-5$. 